\title{
Measurement of sheet resistance of GaN films on a dielectric substrate
}

\author{
Bartlomiej Salski ${ }^{1, a}$, Jerzy Krupka ${ }^{2}$, and Pawel Kopyt ${ }^{1}$ \\ 1 Warsaw University of Technology, Institute of Radioelectronics, Warsaw, Poland \\ 2 Warsaw University of Technology, Institute of Microelectronics and Optoelectronics, Warsaw, Poland
}

Received: 15 April 2014

Published online: 27 August 2014

(c) The Author(s) 2014. This article is published with open access at Springerlink.com

\begin{abstract}
The sheet resistance of gallium nitride films deposited with metal organic chemical vapor deposition on sapphire is investigated in this paper. Two types of contactless measurement techniques are used for that purpose. The first one is a microwave dielectric resonator technique, widely applicable to the precise electrical characterization of resistive films. Due to a small exposure area, the profile of sheet resistance can be acquired across the whole semiconductor film. Another technique is based on a frequency domain capacitive approach, where the film under test functions as a resistive electrode. The method is supplemented with full-wave electromagnetic simulations as well as equivalent circuit representation. The capacitive method is sensitive mostly to the out-of-plane resistance of the layer. Consequently, the combination of both methods provides the tools for the comprehensive and quantitative evaluation of the quality of the semiconductor film.
\end{abstract}

\section{Introduction}

Gallium nitride (GaN), which belongs to the family of III-V direct bandgap semiconductors, has become a very promising material in modern electronics due to its competitive properties, when compared with silicon or gallium arsenide. One of the major advantages of $\mathrm{GaN}$ is a bandgap $\left(E_{g}=3.4 \mathrm{eV}\right)$, about 3 times larger than in silicon, which makes it suitable for high-power transistors. Moreover, the large bandgap allows developing blue-light-emitting diodes and semiconductor lasers. Such devices typically employ thin films of GaN grown on a substrate like sapphire [1]. Simultaneously with the development of thin GaN films technology, intensive research has been prompted on their characterization, especially on the measurements of their electrical properties, such as doping level, charge carriers mobility and resistivity. The aforementioned measurements cannot be performed employing a well-known four-point probe technique due to the lack of a good electrical contact between the electrodes of the probe and the GaN film.

To avoid that problem, contactless measurement techniques have been developed [2-5]. One of the contactless methods applicable to the measurements of sheet resistance and resistivity of semiconductor wafers and thin semiconducting films deposited on semi-insulating substrates is a microwave dielectric resonator technique [4]. Another method, namely, the time domain capacitive technique [2] does not allow measurements of low- and medium-resistivity materials, so it cannot be directly employed to the measurement of sheet resistance of thin epitaxial GaN films. However, a frequency domain capacitive technique, widely recognized in the electrochemistry of resistive electrodes, where one plate of a measured capacitor is made of a resistive layer under test [6], seems to be useful for such purpose. Therefore, the aim of this paper is to investigate the electrical properties of epitaxial GaN layers deposited on sapphire substrates with the capacitive resistive electrode technique and to compare the measurement results with the microwave dielectric resonator technique.

GaN samples that are used in our investigation were grown by metal organic chemical vapor deposition at the Institute of Electronic Materials Technology in Poland. The evaluation of sheet resistance from capacitive measurements will be undertaken with the aid of full-wave electromagnetic simulations as well as an equivalent circuit representation of the scenario. Section 2 is focused on measurement results obtained with both resonant and capacitive techniques.

\footnotetext{
${ }^{a}$ e-mail: bsalski@ire.pw.edu.pl
} 


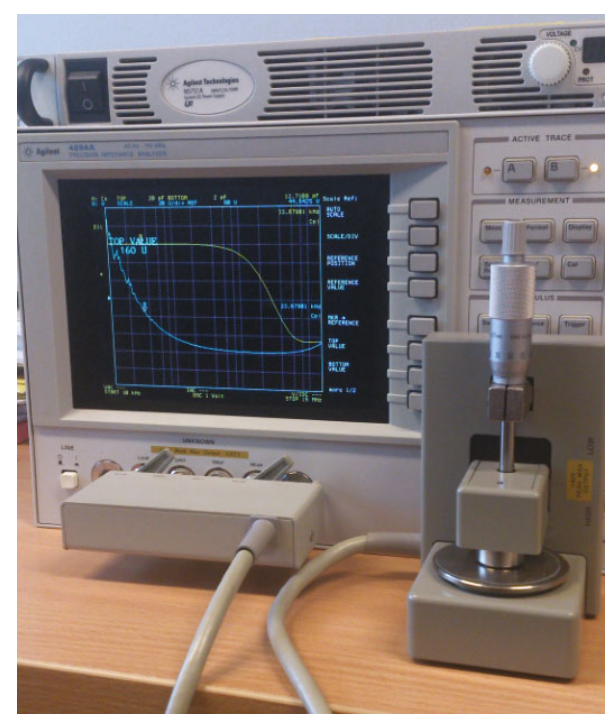

Fig. 1. Measurement setup with a 16451B probe connected to a 4294A Precision Impedance Analyzer via a $5 \mathrm{~T}$ connector. The capacitance (yellow curve) and the quality factor (blue curve) of the probe containing a sample are displayed on the screen of the Impedance Analyzer.

In sect. 3, the evaluation of sheet resistance from capacitive measurements supported with rigorous electromagnetic simulations is discussed. Finally, sect. 4 concentrates on a lumped equivalent circuit representing a capacitive probe introduced in sect. 2, which can be applied to determine the major electric properties of a material under test (MUT).

\section{Measurement setup}

Measurements of GaN layers deposited on a dielectric substrate are undertaken with the aid of a 16451B Agilent dielectric test fixture, which is originally applicable to the evaluation of dielectric constant of solid materials [7]. The probe operates as a parallel-plate capacitor with two flat electrodes, where the MUT is inserted. The probe is connected to a 4294A Precision Impedance Analyzer, which allows measuring the effective capacitance of the MUT (see fig. 1). A five-terminal (5T) connector is applied to enable accurate measurements of impedances spanning from $10 \mathrm{~m} \Omega$ up to $10 \mathrm{M} \Omega$ in the range up to $110 \mathrm{MHz}[8]$.

According to [4], the effective capacitance of a thin resistive film on a dielectric substrate, characterized with the probe which is shown in fig. 1, exhibits relaxation properties, that can be represented with lumped equivalent circuits, depicted in fig. 2. If the film is inhomogeneous and consists of several layers, a multi-pole Debye model, like the one depicted in fig. 2(c), has to be considered. The sheet resistance of the film can be determined provided that the elements of the equivalent circuit are fitted to the measurement data, i.e. capacitance and the quality factor [4].

The diameter of the sapphire substrates is $50.8 \mathrm{~mm}$ and their thickness is about $0.44 \mathrm{~mm}$. The $c$-axis of sapphire is perpendicular to the substrate, so that its permittivity is uniaxially anisotropic with the component parallel to the $c$-axis equal to $\varepsilon_{r z}=11.35$ [9]. The thickness of GaN films is of the order of a few microns. Figure 3 shows the capacitance and the quality factor measured at the center and on the side of two GaN-on-sapphire samples. Although both measurements have a similar characteristic frequency of about $2-3 \mathrm{MHz}$, which is directly related to the diameter of the resistive film functioning as a lossy electrode, the measurement in the GaN center has a much larger slope of the capacitance, whereas the quality factor is about 2 times smaller at the minimum. Such a significant discrepancy between the measurements implies that the GaN layers are inhomogeneous and the most likely reason for it is irregular doping. In addition, tests have shown that none of the measurement results can be accurately approximated with a multi-pole Debye model, unlike the other semiconductor films investigated in [4]. Therefore, additional efforts have to be undertaken to anticipate the equivalent circuit of the scenario and, thus, determine the sheet resistance of the GaN layers.

Subsequently, the profile of sheet resistance is measured with an automated translation stage equipped with a split post-dielectric resonator (SPDR) operating at the frequency of $14.6 \mathrm{GHz}$, which is dedicated to the precise characterization of high-resistivity materials [4]. Figure 4 presents sheet resistance profiles measured on our two samples of GaN on sapphire in two orthogonal directions. It is seen that the resistance is strongly inhomogeneous across each of the samples with the largest values in the middle. It agrees well with the conclusions derived from the capacitive measurements. 


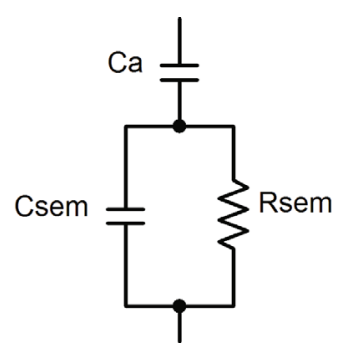

(a)

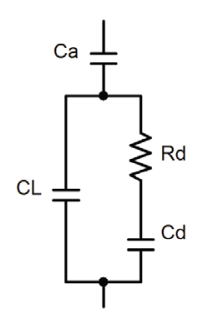

(b)

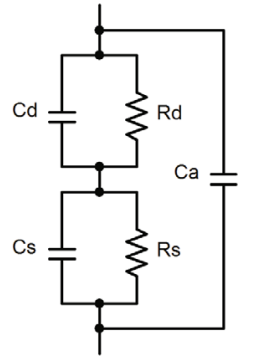

(c)

Fig. 2. Exemplary lumped models representing the effective capacitance of a resistive film deposited on a dielectric substrate characterized with the probe shown in fig. 1 [4].

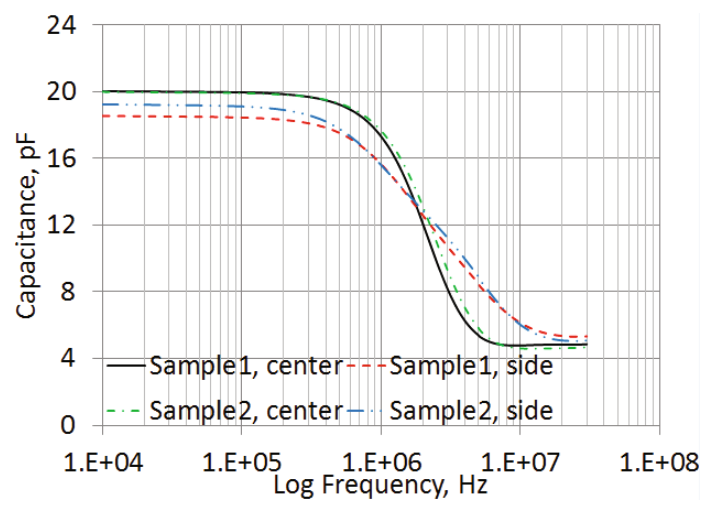

(a)

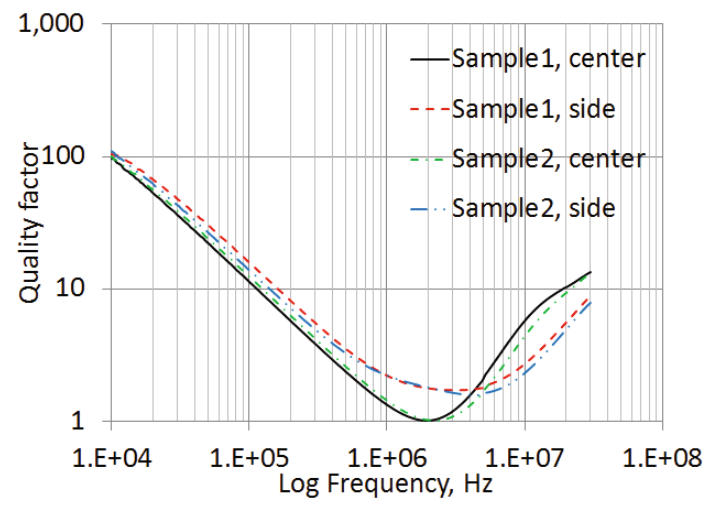

(b)

Fig. 3. (a) Capacitance and (b) quality factor measured in the center and on the side of 2 samples of a GaN layer deposited on sapphire. 


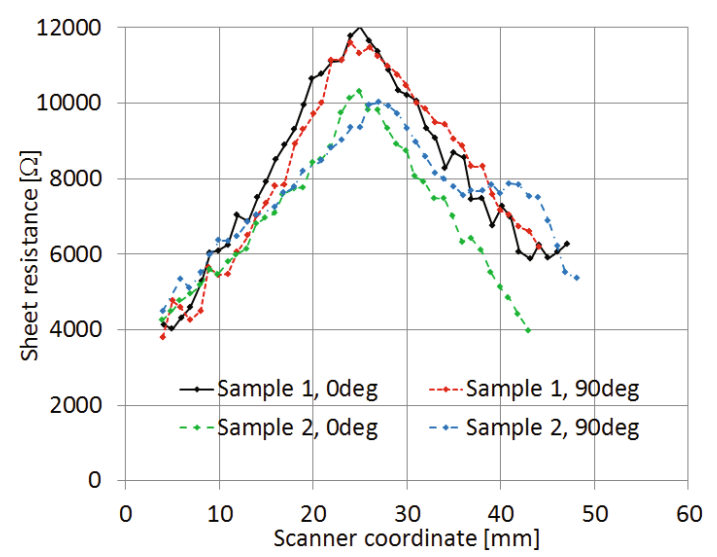

Fig. 4. Sheet resistance profiles of GaN layers deposited on sapphire measured with a split post-dielectric resonator at $f=$ $14.6 \mathrm{GHz}$.

It should be emphasized, however, that the distribution of the sheet resistance shown in fig. 4 cannot be directly applied to the search for an equivalent circuit representing data depicted in fig. 3 . The reasons are at least two. The first is that SPDR measurements are undertaken at a frequency very distant from those considered with the capacitive probe. The second reason, which is even more important, is that measurements with SPDR are performed with the electric field tangential to the GaN film, while the capacitive probe operates with the electric field normal to the film surface.

\section{Electromagnetic modelling}

The measurements obtained with the capacitive probe and shown in fig. 3 can be applied to determine the sheet resistance of GaN films on sapphire. For that purpose, a rigorous electromagnetic model of the measurement setup shown in fig. 1 will be elaborated and computed with a finite-difference time domain (FDTD) method implemented in a commercial software package [10]. The FDTD model should account for the geometry of both the probe and the 5T connector (see fig. 5(a)), which compensates all parasitic elements, in order to accurately represent the measurement. Figure 5(b) depicts the cross-section of the model with the additional guard surrounding the top electrode [7]. The source is represented with lumped impedance connected to one of $50 \Omega$ coaxial cables, while the voltage and current are measured at the terminals of other coaxial cables consisting in the $5 \mathrm{~T}$ connector. Due to axial symmetry of the probe, magnetic symmetry is applied to reduce the size of the FDTD model and alleviate the computational effort of the analysis (see fig. 5(c)). Tests have shown that the field is strongly concentrated between the electrodes and no radiation is observed in the investigated frequency spectrum. Thus, the scenario can be enclosed in a metallic cavity with no visible impact on the accuracy. For simplicity, the cross-section of coaxial cables is assumed to be rectangular but their $50 \Omega$ characteristic impedance is maintained.

As it can be seen in fig. 5(d), a non-equidistant FDTD mesh is applied to efficiently discretize the model. It also depicts a resistive layer deposited on a dielectric substrate and inserted between the electrodes of the probe. Since the dimensions of the scenario are significantly smaller than the shortest wavelength in the considered spectrum, the smallest FDTD cell size is imposed only by the geometry of the probe and not by the numerical dispersion of the FDTD algorithm. Therefore, the increase of the cell size in the regions where no fine geometrical details are present is allowed and highly recommended. The whole FDTD model requires $20 \mathrm{MB}$ of RAM and a single electromagnetic simulation takes about 50 minutes on an NVidia GeForce GTX 580 video card, mainly due to a prohibitively small FDTD time step.

According to the measurement data given in the previous section, the permittivity of sapphire is set to $\varepsilon_{r z}=11.35$. The resistive film is represented with a single layer of FDTD cells, the thickness of which is $h=10 \mu \mathrm{m}$. Consequently, the equivalent conductivity of the layer is $\sigma=\left(h R_{s q}\right)^{-1}=10 \mathrm{~S} / \mathrm{m}$, where $R_{s q}$ stands for sheet resistance of the film.

Figure 6 (a) shows an electric field distribution calculated with FDTD at $10 \mathrm{MHz}$. It can be noticed that the electric field is strongly concentrated between the electrodes, where the MUT is located. The electric field is also visible in two coaxial cables connected to the source and voltage probes. On the contrary, no electric field can be observed in the left coaxial line since the impedance of the current probe is negligible. The exemplary spectral characteristics of voltage and current, which allow the calculation of an effective capacitance of the probe

$$
C_{e f f}(\omega)=\frac{I(\omega)}{j \omega U(\omega)},
$$

are shown in fig. 6(b). 


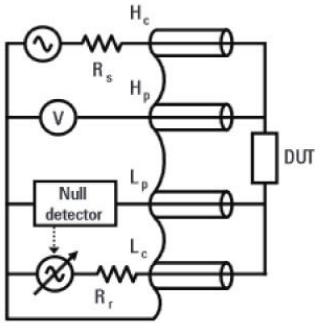

(a)

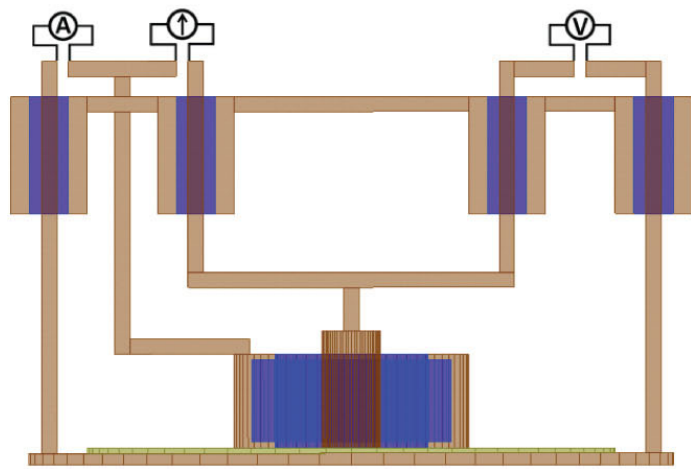

(b)

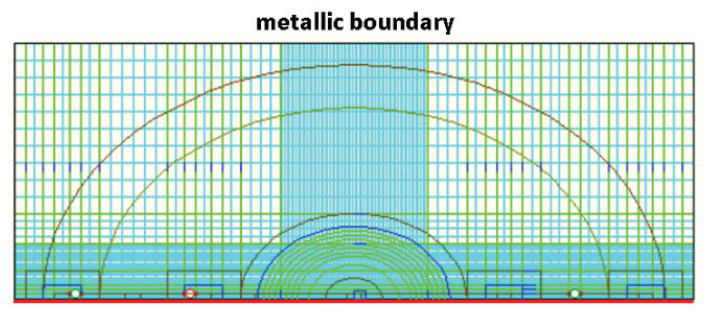

magnetic symmetry

(c)

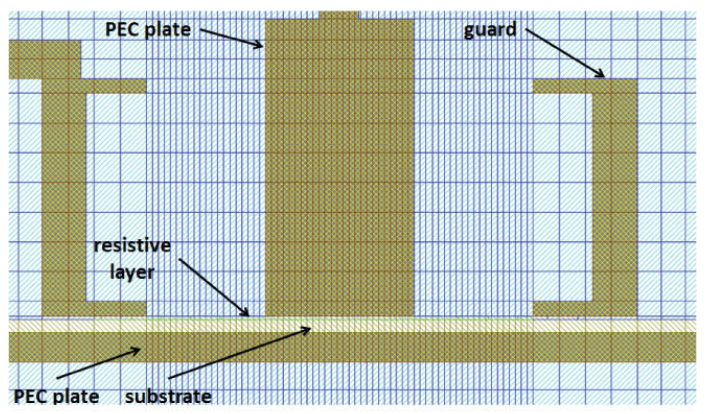

(d)

Fig. 5. (b) FDTD model of a 16451B Agilent dielectric test fixture with (a) a 5T connector. (c) 2D cross-section of the FDTD model truncated with magnetic symmetry. (d) View of the FDTD mesh in the neighborhood of electrodes.

The effective capacitance obtained from the FDTD analysis using eq. (1) is compared with measurement data as shown in fig. 7. The best fitting with measurements shown in fig. 3 are obtained for the sheet resistance of the GaN film set to $R_{s q}=10 \mathrm{k} \Omega / \mathrm{sq}$, which is close to the results obtained with SPDR on the side of the GaN samples (see fig. 4). It can be also noticed that the FDTD results are very well fitted to a Debye model $\left(\varepsilon_{s}=22.3, \varepsilon_{\text {inf }}=4.8, \tau=79.58 \mathrm{~ns}\right)$, which is in a good agreement with experiments undertaken for homogeneous semiconductor films characterized with the capacitive probe [4]. However, the Cole-Cole plots shown in fig. 7(c) indicate that the measured GaN-on-sapphire samples do not behave like the Debye relaxation model, since the shape of the complex functions is not circular. According to [6], a deformed shape of characteristics usually indicates a porous structure of the lossy electrode. Since 


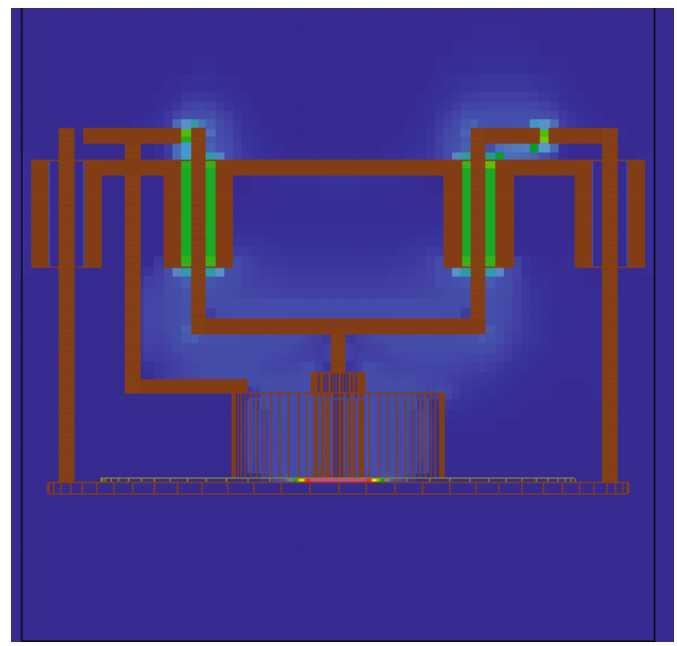

(a)

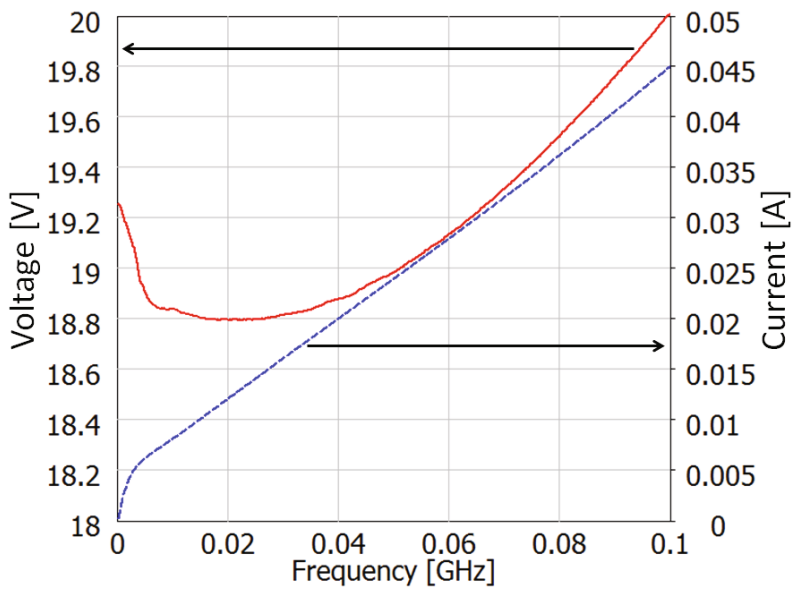

(b)

Fig. 6. (a) Distribution of an electric field at $10 \mathrm{MHz}$ computed in the FDTD model presented in fig. 5 and (b) exemplary spectral characteristics of voltage and current.

the investigated GaN films are epitaxially grown on sapphire, the porosity does not occur. The unusual shape of the Cole-Cole plots results rather from inhomogeneous doping of gallium nitride, which is one the challenges to be overcome in the metal organic chemical vapor deposition.

In the case of the measurement on the side of the sample, the plot can be represented with a Havriliak-Negami (H-N) dispersion model, which is typical for diffusion processes with asymmetrically distributed relaxation frequencies [11]:

$$
C(\omega)=C_{\infty}+\frac{C_{s}-C_{\infty}}{\left(1+(j \omega \tau)^{1-\alpha}\right)^{\beta}},
$$

where $C_{\infty}=5 \mathrm{pF}, C_{S}=18.5 \mathrm{pF}, \tau=0.118 \mu \mathrm{s}, \alpha=-0.075, \beta=0.9$. The measurement at the center of the sample, where doping inhomogeneity is the largest, has a significantly magnified imaginary part of the effective capacitance, so that the Havriliak-Negami model becomes inappropriate.

The experimental results addressed in this section have shown that GaN layers, grown on sapphire by the metal organic chemical vapor deposition, cannot be represented with the single-pole Debye relaxation model. It has been rigorously proven with the aid of the full-wave FDTD analysis, that the homogeneous lossy electrode behaves according to the Debye model. Electromagnetic simulations have also depicted the approximate value of the sheet resistance of the GaN film, which is about $10 \mathrm{k} \Omega / \mathrm{sq}$. If doping inhomogeneity is not significant, as in the case of the measurements on the side of the sample, spectral characteristics can be represented with the Havriliak-Negami dispersion model. 


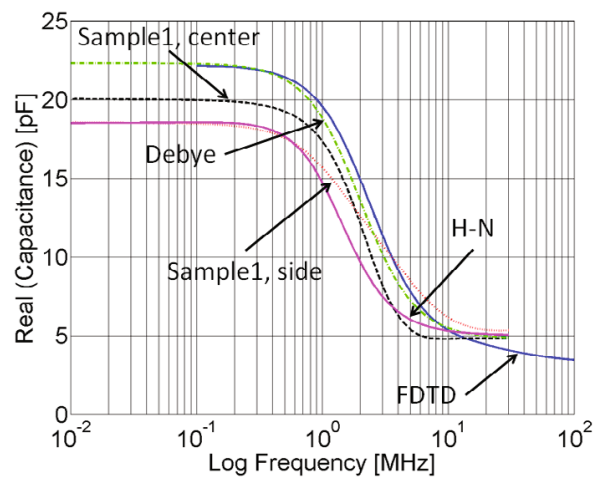

(a)

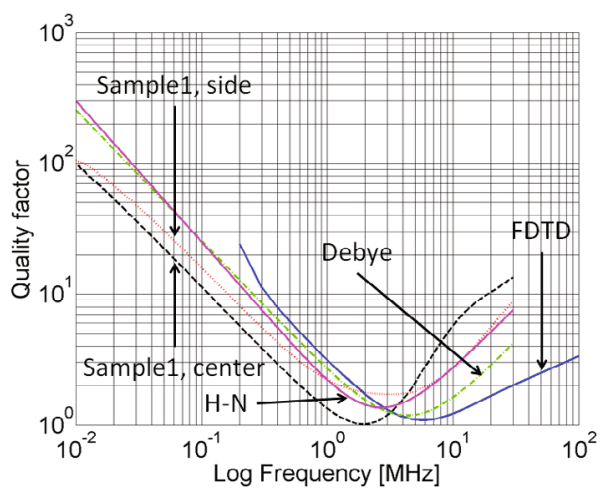

(b)

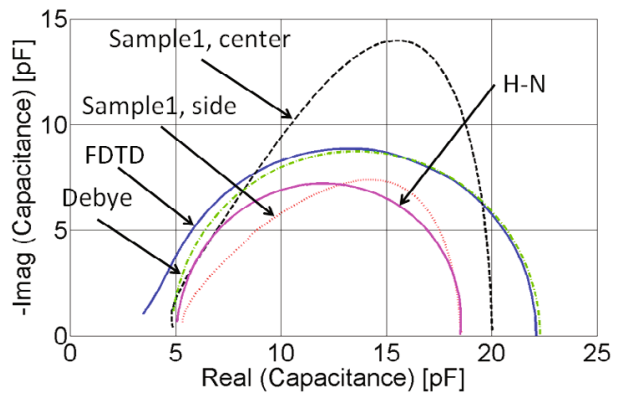

(c)

Fig. 7. (a) Capacitance, (b) quality factor and (c) Cole-Cole plots of data measured in the center and on the side of 2 samples of a GaN layer deposited on sapphire, compared with the FDTD results and fitted to the Debye and Havriliak-Negami models.

\section{Equivalent circuit}

The next step in the investigation is to find a lumped equivalent circuit approximating the measured data, which may help deriving essential conclusions about the structure of the GaN films deposited on sapphire. As will be shown, the advantage of having the equivalent circuit over analytical models, like the Havriliak-Negami model successfully applied in the previous section, lies in the possibility of associating lumped elements of the model with particular phenomena occurring during the measurement. Several types of circuits have been tested, but only the one shown in fig. 8(a) provides the effective capacitance, which is in relatively good agreement with measurement results. The equivalent circuit consists of a dispersive branch shunted with a capacitor $C_{2}=5.52 \mathrm{pF}$, which represents the high frequency capacitance of the $16451 \mathrm{~B}$ probe, which can be estimated by the following formula:

$$
C_{2} \cong \varepsilon_{0} \varepsilon_{r} \frac{\pi R^{2}}{h}=4.48 \mathrm{pF},
$$

where $R=2.5 \mathrm{~mm}$ is the radius of the upper plate of the probe, and $h=0.44 \mathrm{~mm}$ stands for the thickness of the substrate. 


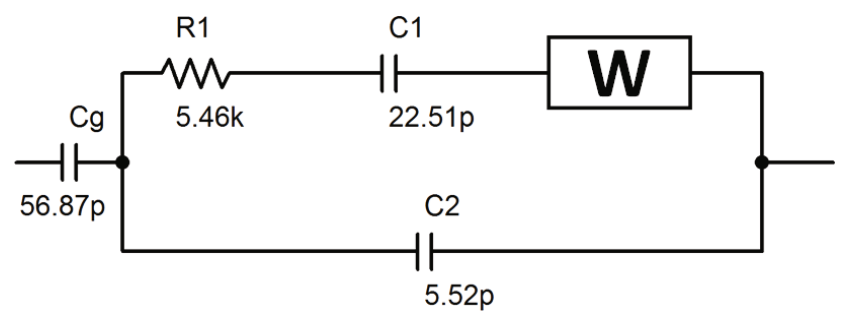

(a)

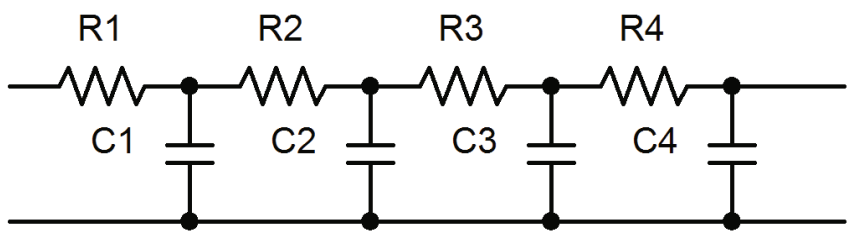

(b)

Fig. 8. Equivalent circuits of (a) GaN on sapphire and (b) a distributed RC network representing Warburg impedance $Z_{W}$.

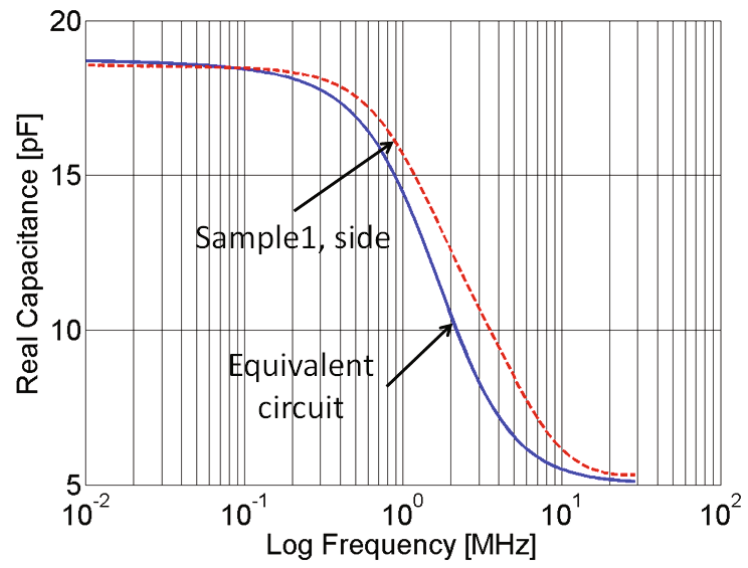

(a)

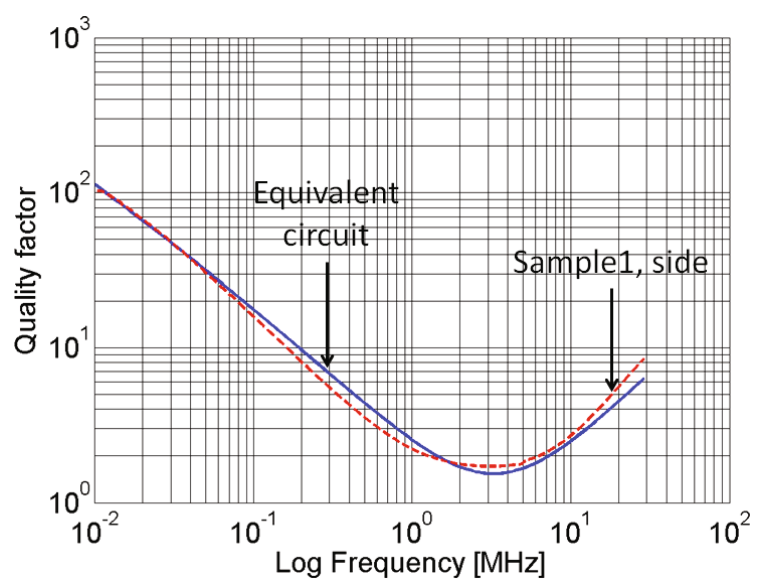

(b)

Fig. 9. (a) Capacitance and (b) quality factor measured on the side of a GaN layer deposited on sapphire compared with an equivalent circuit shown in fig. 9(a). 
The capacitor $C_{g}=56.87 \mathrm{pF}$ represents a small air gap, which frequently occurs between the upper electrode and the GaN layer. It can be thus estimated that the effective thickness of such an air gap is about $3 \mu \mathrm{m}$. The dispersive branch in fig. 8(a) is widely applicable in the impedance spectroscopy of porous/inhomogeneous electrodes, which can occur in several electrochemical processes [6]. The Warburg impedance $Z_{w}$ :

$$
Z_{W}=\frac{R_{W}}{(j \omega)^{\alpha}}
$$

consisting in that branch, accounts for a distributed nature of the lossy electrode, where $R_{W}=1.2 \mathrm{e} 6, \alpha=0.45$. Figure 8(b) shows an RC network, the characteristic impedance of which can be represented with eq. (4).

Figure 9 depicts the comparison of measurement data with the proposed equivalent circuit and it can be noticed that much better agreement, when compared with fig. 7 , is obtained. The lumped resistance $R_{1}=5.46 \mathrm{k} \Omega$ agrees very well with microwave measurements of sheet resistance on the side of the GaN samples (see fig. 4). Unfortunately, all the investigated types of equivalent circuits are not useful to represent accurately the measurement results at the center of the two characterized GaN samples, which can be the subject of further studies.

\section{Conclusion}

It has been shown in this paper that the sheet resistance of gallium nitride deposited on sapphire with metal organic chemical vapor deposition can be characterized with a split post-dielectric resonator operating at microwave frequencies. Due to a relatively small exposure area in SPDRs operating at $14.6 \mathrm{GHz}$, the profile of sheet resistance can be acquired across the whole sample under test. On the contrary, the measurement with a parallel-plate capacitive probe is much less localized as the whole GaN layer functions as a lossy and distributed electrode. However, it gives deeper insight into microscopic properties of the layer and, moreover, it is sensitive mostly to the out-of-plane resistance of the layer. Consequently, the combination of both methods provides the tools for the comprehensive and quantitative evaluation of the film quality.

Part of this work was funded by the Polish National Centre for Research and Development under GRAF-TECH/ NCBR/09/07/2013 contract and by the European Union in the framework of European Social Fund through the Warsaw University of Technology Development Programme.

Open Access This is an open access article distributed under the terms of the Creative Commons Attribution License (http://creativecommons.org/licenses/by/4.0), which permits unrestricted use, distribution, and reproduction in any medium, provided the original work is properly cited.

\section{References}

1. P. Russel, SiC, sapphire and GaN materials status into Opto and RF businesses, in Proceedings of the CS MANTECH Conference, Vancouver, British Columbia, Canada, April 24-27 (2006) pp. 231-232.

2. R. Stibal, J. Windscheif, W. Jantz, Semicond. Sci. Technol. 6, 955 (1991).

3. G.L. Miller, D.A.H. Robinson, J.D. Wiley, Rev. Sci. Instrum. 47, 799 (1976).

4. J. Krupka, Meas. Sci. Technol. 24, 062001 (2013).

5. J.R. MacDonald, Impedence Spectroscopy-Emphasizing Solid Materials and Systems (Wiley-Interscience, John Wiley and Sons, 1987).

6. M. Schlesinger, Modern Aspects of Electrochemistry (Springer, 2009).

7. 16451B Dielectric Test Fixture, available at http://www.home.agilent.com.

8. Agilent Impedance Measurement Handbook. A guide to measurement technology and techniques, 4th edition, available at http://www .home.agilent.com.

9. J. Krupka, K. Derzakowski, A. Abramowicz, M.E. Tobar, R.G. Geyer, IEEE Trans. Microwave Theory Tech. 47, 752 (1999).

10. QuickWave-3D, QWED Sp. z o. o., 1997-2014, available at: http://www.qwed.com.pl.

11. S. Havriliak, S. Negami, Polymer 8, 161 (1967). 